\title{
Low weight perfect matchings
}

\author{
Stefan Ehard* Elena Mohr Dieter Rautenbach \\ Institute of Optimization and Operations Research \\ Ulm University \\ Germany \\ \{stefan.ehard, elena.mohr, dieter.rautenbach\}@uni-ulm.de
}

Submitted: Nov 3, 2020; Accepted: Dec 8, 2020; Published: Dec 24, 2020

(c) The authors. Released under the CC BY-ND license (International 4.0).

\begin{abstract}
Answering a question posed by Caro, Hansberg, Lauri, and Zarb, we show that for every positive integer $n$ and every function $\sigma: E\left(K_{4 n}\right) \rightarrow\{-1,1\}$ with $\sigma\left(E\left(K_{4 n}\right)\right)=0$, there is a perfect matching $M$ in $K_{4 n}$ with $\sigma(M)=0$. Strengthening the consequence of a result of Caro and Yuster, we show that for every positive integer $n$ and every function $\sigma: E\left(K_{4 n}\right) \rightarrow\{-1,1\}$ with $\left|\sigma\left(E\left(K_{4 n}\right)\right)\right|<n^{2}+11 n+2$, there is a perfect matching $M$ in $K_{4 n}$ with $|\sigma(M)| \leqslant 2$. Both these results are best possible.
\end{abstract}

Mathematics Subject Classifications: 05C22, 05C70

\section{Introduction}

In [2] Caro, Hansberg, Lauri, and Zarb considered connected graphs $G$ together with a function $\sigma: E(G) \rightarrow\{-1,1\}$ labeling the edges of $G$ with -1 or +1 , and they studied conditions that imply the existence of different types of spanning trees $T$ with

$$
|\sigma(E(T))|=\left|\sum_{e \in E(T)} \sigma(e)\right| \leqslant 1,
$$

where, as usual, for a set $E$ of edges, $\sigma(E)$ is just the sum of $\sigma(e)$ over all $e$ in $E$. As a variation of this problem, they ask whether, for every positive integer $n$ and every labeling $\sigma: E\left(K_{4 n}\right) \rightarrow\{-1,1\}$ of the edges of the complete graph $K_{4 n}$ of order $4 n$ with $\sigma\left(E\left(K_{4 n}\right)\right)=0$, there is a perfect matching $M$ in $K_{4 n}$ with $\sigma(M)=0$. We answer their question in the affirmative.

${ }^{*}$ Funded by the Deutsche Forschungsgemeinschaft (DFG, German Research Foundation) - 388217545. 
Theorem 1. For every positive integer $n$ and every function $\sigma: E\left(K_{4 n}\right) \rightarrow\{-1,1\}$ with $\sigma\left(E\left(K_{4 n}\right)\right)=0$, there is a perfect matching $M$ in $K_{4 n}$ with $\sigma(M)=0$.

In order to put our result into some wider perspective, we briefly discuss the notion of local amoebas studied in $[2,3]$ : A graph $G$ of order $n$ is a local amoeba if, for every two isomorphic copies $H$ and $H^{\prime}$ of $G$ in $K_{n}$, there is a sequence $G_{0}, \ldots, G_{k}$ of isomorphic copies of $G$ in $K_{n}$ such that $H=G_{0}, H^{\prime}=G_{k}$, and each $G_{i+1}$ arises from $G_{i}$ by an edge-replacement, that is, $E\left(G_{i+1}\right)=\left(E\left(G_{i}\right) \backslash\{e\}\right) \cup\left\{e^{\prime}\right\}$ for some $e \in E\left(G_{i}\right)$ and $e^{\prime} \in$ $E\left(K_{n}\right) \backslash E\left(G_{i}\right)$. Note that a path $P_{n}$ is an example of a local amoeba, and that local amoebas are defined by an exchange property that very closely resembles the well-known exchange property of the bases of a matroid: For every two bases $B$ and $B^{\prime}$ of a matroid, and for every $e^{\prime} \in B^{\prime} \backslash B$, there is some $e \in B \backslash B^{\prime}$ such that $(B \backslash\{e\}) \cup\left\{e^{\prime}\right\}$ is again a basis of the matroid. Now, perfect matchings of a complete graph are a very natural example of spanning subgraphs that are no local amoebas, and for which, consequently, the machinery developed in [2] fails. In fact, perfect matchings have the following slightly weaker exchange property: If $M$ and $M^{\prime}$ are two distinct perfect matchings in $K_{n}$, then, considering an $M$ - $M^{\prime}$-alternating cycle defined by their symmetric difference $M \Delta M^{\prime}$, it follows that there are two edges $e_{1}, e_{2}$ in $M$, one edge $e_{1}^{\prime}$ in $M^{\prime}$, and one further edge $e_{2}^{\prime}$, which might not belong to $M \cup M^{\prime}$, such that $M^{\prime \prime}=\left(M \backslash\left\{e_{1}, e_{2}\right\}\right) \cup\left\{e_{1}^{\prime}, e_{2}^{\prime}\right\}$ is a perfect matching in $K_{n}$ for which $\left|M^{\prime} \Delta M^{\prime \prime}\right|$ is strictly smaller than $\left|M^{\prime} \Delta M\right|$, that is, one can transform $M$ into $M^{\prime}$ by a sequence of exchange operations removing and adding two edges, and not just one, at every step.

Under the hypothesis of Theorem 1, the existence of a perfect matching $M$ in $K_{4 n}$ with $|\sigma(M)| \leqslant 2$ already follows from more general results due to Caro and Yuster, cf. Theorem 1.1 in [4]. More precisely, Caro and Yuster showed that the weaker hypothesis $\left|\sigma\left(E\left(K_{4 n}\right)\right)\right| \leqslant 2(4 n-1)$ suffices for the existence of such a perfect matching $M$ with $|\sigma(M)| \leqslant 2$. As observed in [2], for infinitely many positive integers $n$, there are functions $\sigma: E\left(K_{4 n}\right) \rightarrow\{-1,1\}$ with $\sigma\left(E\left(K_{4 n}\right)\right)=4 \sqrt{n}-2$ such that $\sigma(M) \neq 0$ for every perfect matching $M$ in $K_{4 n}$. Slightly modifying their construction, we obtain the following proposition, which implies that Theorem 1 is best possible for infinitely many values of $n$.

Proposition 2. For infinitely many positive integers n, there is a function $\sigma: E\left(K_{4 n}\right) \rightarrow$ $\{-1,1\}$ with $\sigma\left(E\left(K_{4 n}\right)\right)=2$ such that $\sigma(M) \neq 0$ for every perfect matching $M$ in $K_{4 n}$.

Considering the construction in the proof of Proposition 2 suggests that zero weight perfect matchings are excluded rather by parity reasons than by the value of the imbalance $\left|\sigma\left(E\left(K_{4 n}\right)\right)\right|$ of $\sigma$. We confirm this with our second main result showing that much weaker conditions on the imbalance imply the existence of low weight perfect matchings.

Theorem 3. For all positive integers $n$ and $k$ such that $k \geqslant 2$, and every function $\sigma: E\left(K_{4 n}\right) \rightarrow\{-1,1\}$ with $\left|\sigma\left(E\left(K_{4 n}\right)\right)\right|<n(n-1)+k(6 n-1)+k^{2}$, there is a perfect matching $M$ in $K_{4 n}$ with $|\sigma(M)| \leqslant 2 k-2$.

For $k=2$, Theorem 3 implies the following strengthening of the above-mentioned consequence of the result of Caro and Yuster. 
Corollary 4. For every positive integer $n$ and every function $\sigma: E\left(K_{4 n}\right) \rightarrow\{-1,1\}$ with $\left|\sigma\left(E\left(K_{4 n}\right)\right)\right|<n^{2}+11 n+2$, there is a perfect matching $M$ in $K_{4 n}$ with $|\sigma(M)| \leqslant 2$.

Both, Theorem 3 and, hence, also Corollary 4 are best possible. If, for instance, $\sigma: E\left(K_{4 n}\right) \rightarrow\{-1,1\}$ is such that the graph $\left(V\left(K_{4 n}\right), \sigma^{-1}(1)\right)$ consists of a clique of order $3 n+2$ and $n-2$ isolated vertices, then $|\sigma(M)| \geqslant 4$ for every perfect matching $M$ in $K_{4 n}$ while $\left|\sigma\left(E\left(K_{4 n}\right)\right)\right|=n^{2}+11 n+2$. In conjunction, Theorem 1, Proposition 2, and Corollary 4 imply an interesting behavior: If $n$ is a positive integer and $\sigma$ is a \pm 1 -labeling of the edges of $K_{4 n}$, then, in order to force the existence of a zero weight perfect matching, one needs to require zero imbalance, that is, $\left|\sigma\left(E\left(K_{4 n}\right)\right)\right|=0$, while $|\sigma(M)|>2$ for every perfect matching $M$ in $K_{4 n}$ already forces a quadratic imbalance, that is, $\left|\sigma\left(E\left(K_{4 n}\right)\right)\right|$ is at least quadratic in $n$.

All proofs are given in the next section.

For a survey concerning related results, we refer the reader to [1] and the introduction of $[2]$.

\section{Proofs}

We start with the proof of our first main result.

Proof of Theorem 1. We suppose, for a contradiction, that $\sigma: E\left(K_{4 n}\right) \rightarrow\{-1,1\}$ is such that $\sigma\left(E\left(K_{4 n}\right)\right)=0$ but that $\sigma(M) \neq 0$ for every perfect matching $M$ in $K_{4 n}$. First, we consider the case $n=1$. The edge set of $K_{4}$ is the union of three edge-disjoint perfect matchings $M_{1}, M_{2}$, and $M_{3}$. Since $\sigma\left(M_{i}\right) \neq 0$ for every $i$, we obtain $\sigma\left(M_{i}\right) \in\{-2,2\}$, which implies the contradiction $\sigma\left(E\left(K_{4 n}\right)\right)=\sigma\left(M_{1}\right)+\sigma\left(M_{2}\right)+\sigma\left(M_{3}\right) \neq 0$. Hence, we may assume that $n \geqslant 2$. We call an edge $e$ a plus-edge if $\sigma(e)=1$, and a minus-edge if $\sigma(e)=-1$. Since $\sigma\left(E\left(K_{4 n}\right)\right)=0$,

there are exactly $\frac{1}{2}\left(\begin{array}{c}4 n \\ 2\end{array}\right)=4 n^{2}-n$ plus-edges and minus-edges in $K_{4 n}$, respectively.

For a matching $M$, we denote by $M^{+}$and $M^{-}$the sets of plus-edges and minus-edges in $M$, respectively. We choose a perfect matching $M$ in $K_{4 n}$ such that $|\sigma(M)|$ is as small as possible. Possibly replacing $\sigma$ with $-\sigma$, we may assume that $\sigma(M)>0$. Since $M$ contains $2 n$ edges, $\sigma(M)$ is even, which implies $\sigma(M) \geqslant 2$.

We start with some easy observations.

Claim 5. For every two edges e in $M^{+}$and $f$ in $M^{-}$, there are no two disjoint minusedges between $e$ and $f$. In particular, there are at most two minus-edges between e and $f$.

Proof of Claim 5. If there are two disjoint minus-edges $e^{\prime}$ and $f^{\prime}$ between two edges $e \in$ $M^{+}$and $f \in M^{-}$, then the perfect matching $N=(M \backslash\{e, f\}) \cup\left\{e^{\prime}, f^{\prime}\right\}$ satisfies $0 \leqslant$ $\sigma(N)=\sigma(M)-2<\sigma(M)$, contradicting the choice of $M$.

Claim 6. There are two edges e and $f$ in $M^{+}$such that there exists a minus-edge between $e$ and $f$. 
Proof of Claim 6. Suppose, for a contradiction, that there is no minus-edge between vertices in $V\left(M^{+}\right)$; that is, more formally, the subgraph of $K_{4 n}$ induced by the set $V\left(M^{+}\right)$ of vertices that are incident with a plus-edge from $M$ contains no minus-edge of $K_{4 n}$. For $m^{+}=\left|M^{+}\right|$, we have $m^{+} \geqslant n+1$. By Claim 5 , at least half the $2 m^{+}\left(4 n-2 m^{+}\right)$edges between $V\left(M^{+}\right)$and $V\left(M^{-}\right)$are plus-edges, and, hence, the total number of plus-edges is at least

$$
\left(\begin{array}{c}
2 m^{+} \\
2
\end{array}\right)+\frac{1}{2} \cdot 2 m^{+}\left(4 n-2 m^{+}\right)=(4 n-1) m^{+} \geqslant(4 n-1)(n+1)>4 n^{2}-n,
$$

contradicting (1).

Claim 7. For every two edges $u_{1} u_{2}$ and $v_{1} v_{2}$ in $M^{+}$, if $u_{1} v_{1}$ is a minus-edge, then $u_{2} v_{2}$ is also a minus-edge. Furthermore, $\sigma(M)=2$.

Proof of Claim \%. If $u_{1} u_{2}$ and $v_{1} v_{2}$ are two edges in $M^{+}$such that $u_{1} v_{1}$ is a minus-edge and $u_{2} v_{2}$ is a plus-edge, then the perfect matching $N=\left(M \backslash\left\{u_{1} u_{2}, v_{1} v_{2}\right\}\right) \cup\left\{u_{1} v_{1}, u_{2} v_{2}\right\}$ satisfies $0 \leqslant \sigma(N)=\sigma(M)-2<\sigma(M)$, contradicting the choice of $M$. This implies the first part of the statement. Now, suppose, for a contradiction, that $\sigma(M)>2$. Since $\sigma(M)$ is even, we have $\sigma(M) \geqslant 4$. By Claim 6, there are two edges $u_{1} u_{2}$ and $v_{1} v_{2}$ in $M^{+}$ such that $u_{1} v_{1}$ and $u_{2} v_{2}$ are both minus-edges. Now, the perfect matching $N$ as above satisfies $0 \leqslant \sigma(N)=\sigma(M)-4<\sigma(M)$, contradicting the choice of $M$. This completes the proof of the claim.

Since $\sigma(M)=2$, the matching $M$ contains exactly $n+1$ plus-edges and $n-1$ minusedges. We call a perfect matching $N$ in $K_{4 n} \operatorname{good}$ if $\sigma(N)=\sigma(M)$. If $N$ is a good matching, then an edge $e^{+}$in $N^{+}$is called special if there is no minus-edge between vertices in $V\left(N^{+} \backslash\left\{e^{+}\right\}\right)$, that is, all minus-edges between vertices in $V\left(N^{+}\right)$are adjacent with $e^{+}$.

We distinguish the following two cases.

Case 1. Every good matching contains a special edge.

Let $e^{+}$be a special edge in $M$.

Claim 8. For every edge e in $M^{+} \backslash\left\{e^{+}\right\}$, there exist only minus-edges between $e^{+}$and $e$.

Proof of Claim 8. Suppose, for a contradiction, that there is a plus-edge between $e^{+}$and some edge $e$ in $M^{+} \backslash\left\{e^{+}\right\}$. By Claim 7, there are at least two plus-edges between $e^{+}$ and $e$. Since $e^{+}$is special, it follows that there are at most $4 n-2$ minus-edges between vertices in $V\left(M^{+}\right)$. Therefore, by Claim 5 , the total number of plus-edges is at least

$$
\left(\begin{array}{c}
2 n+2 \\
2
\end{array}\right)-(4 n-2)+\frac{1}{2} \cdot(2 n+2)(2 n-2)=4 n^{2}-n+1,
$$

contradicting (1). 
Claim 9. There is no plus-edge between vertices in $V\left(M^{-}\right)$. Furthermore, there is an edge $e^{-}$in $M^{-}$such that there are exactly three plus edges between $e^{+}$and $e^{-}$, and, for every two edges $e$ in $M^{+}$and $e^{\prime}$ in $M^{-}$with $\left(e, e^{\prime}\right) \neq\left(e^{+}, e^{-}\right)$, there are exactly two plus-edges between $e$ and $e^{\prime}$.

Proof of Claim 9. By Claim 8, there are exactly $4 n$ minus-edges between vertices in $V\left(M^{+}\right)$, and, hence, exactly $\left(\begin{array}{c}2 n+2 \\ 2\end{array}\right)-4 n$ plus-edges between vertices in $V\left(M^{+}\right)$. By Claim 5, there are at least $\frac{1}{2} \cdot(2 n+2)(2 n-2)$ plus-edges between $V\left(M^{+}\right)$and $V\left(M^{-}\right)$. Since

$$
\left(\left(\begin{array}{c}
2 n+2 \\
2
\end{array}\right)-4 n\right)+\frac{1}{2} \cdot(2 n+2)(2 n-2)=4 n^{2}-n-1
$$

observation (1) implies the existence of exactly one further plus-edge not yet accounted for.

Suppose, for a contradiction, that $V\left(M^{-}\right)$contains a plus-edge $f_{1}$, that is, there are two edges $e_{1}$ and $e_{2}$ in $M^{-}$such that $f_{1}$ lies between $e_{1}$ and $e_{2}$. Let $f_{2}$ be the edge between $e_{1}$ and $e_{2}$ that is disjoint from $f_{1}$. Since, by (1), $f_{1}$ is the only plus-edge between vertices in $V\left(M^{-}\right)$, it follows that $f_{2}$ is a minus-edge. Let $e_{3}$ be in $M^{+} \backslash\left\{e^{+}\right\}$, and let $f_{3}$ and $f_{4}$ be disjoint minus-edges between $e_{3}$ and $e^{+}$. Now, the perfect matching $N=\left(M \backslash\left\{e^{+}, e_{1}, e_{2}, e_{3}\right\}\right) \cup\left\{f_{1}, f_{2}, f_{3}, f_{4}\right\}$ satisfies $\sigma(N)=\sigma(M)-2=0$, contradicting the choice of $M$.

It follows that there is no plus-edge between vertices in $V\left(M^{-}\right)$and that there are exactly $\frac{1}{2} \cdot(2 n+2)(2 n-2)+1$ plus-edges between $V\left(M^{+}\right)$and $V\left(M^{-}\right)$. By Claim 5 , this implies that there is an edge $\hat{e}$ in $M^{+}$and an edge $e^{-}$in $M^{-}$such that there are exactly three plus edges between $\hat{e}$ and $e^{-}$, and, for every two edges $e$ in $M^{+}$and $e^{\prime}$ in $M^{-}$with $\left(e, e^{\prime}\right) \neq\left(\hat{e}, e^{-}\right)$, there are exactly two plus-edges between $e$ and $e^{\prime}$. In order to complete the proof of the claim, it remains to show that $\hat{e}=e^{+}$. Suppose, for a contradiction, that $\hat{e} \neq e^{+}$. Since $\left|M^{+}\right|=n+1 \geqslant 3$, there is an edge $e_{1}$ in $M^{+} \backslash\left\{e^{+}, \hat{e}\right\}$. Let $f_{1}$ and $f_{2}$ be two disjoint minus-edges between $e^{+}$and $e_{1}$, and let $f_{3}$ and $f_{4}$ be two disjoint plus-edges between $\hat{e}$ and $e^{-}$. The perfect matching $N=\left(M \backslash\left\{e^{+}, \hat{e}, e^{-}, e_{1}\right\}\right) \cup\left\{f_{1}, f_{2}, f_{3}, f_{4}\right\}$ satisfies $\sigma(N)=\sigma(M)-2=0$, contradicting the choice of $M$. Hence, $\hat{e}=e^{+}$, which completes the proof of the claim.

Let $f^{+}$and $f^{-}$be two disjoint edges between $e^{+}$and $e^{-}$such that $f^{+}$is a plus-edge and $f^{-}$is a minus-edge. The perfect matching $N=\left(M \backslash\left\{e^{+}, e^{-}\right\}\right) \cup\left\{f^{+}, f^{-}\right\}$satisfies $\sigma(N)=\sigma(M)$, that is, $N$ is good. By the assumption in Case $1, N$ contains a special edge. Since there are exactly three plus-edges between $f^{+}$in $N^{+}$and $f^{-}$in $N^{-}$, Claim 9 implies that $f^{+}$is a special edge in $N$. If $u^{-}$is such that $\left\{u^{-}\right\}=e^{-} \cap f^{+}$, then Claim 8 implies that there are only minus-edges between $u^{-}$and $V\left(M^{+} \backslash\left\{e^{+}\right\}\right)=V\left(N^{+} \backslash\left\{f^{+}\right\}\right)$. Let $f_{1}$ be the plus-edge between $e^{+}$and $e^{-}$that is not incident with $u^{-}$. Let $e \in M^{+} \backslash\left\{e^{+}\right\}$. Let $f_{2}$ and $f_{3}$ be two disjoint edges between $e^{+} \cup e^{-}$and $e$ that are disjoint from $f_{1}$, in particular, $f_{2}$ and $f_{3}$ are both minus-edges. Now, the perfect matching $M^{\prime}=\left(M \backslash\left\{e^{+}, e^{-}, e\right\}\right) \cup\left\{f_{1}, f_{2}, f_{3}\right\}$ satisfies $\sigma\left(M^{\prime}\right)=\sigma(M)-2=0$, contradicting the choice of $M$, which completes the proof in this case. 
Case 2. Some good matching contains no special edge.

In this case, we may assume that the good matching $M$ chosen at the beginning of the proof contains no special edge.

First, suppose, for a contradiction, that there are two edges $e_{1}$ in $M^{+}$and $e_{2}$ in $M^{-}$such that there are two disjoint plus-edges $f_{1}$ and $f_{2}$ between $e_{1}$ and $e_{2}$. Since $e_{1}$ is not a special edge in $M$, Claim 7 implies the existence of two edges $e_{3}$ and $e_{4}$ both in $M^{+} \backslash\left\{e_{1}\right\}$ such that there are two disjoint minus-edges $f_{3}$ and $f_{4}$ between $e_{3}$ and $e_{4}$. Now, the perfect matching $N=\left(M \backslash\left\{e_{1}, e_{2}, e_{3}, e_{4}\right\}\right) \cup\left\{f_{1}, f_{2}, f_{3}, f_{4}\right\}$ satisfies $\sigma(N)=\sigma(M)-2=0$, contradicting the choice of $M$. Hence, there are no two such edges as $e_{1}$ and $e_{2}$. Together with Claim 5 this implies that there are exactly $\frac{1}{2} \cdot(2 n+2)(2 n-2)=2(n+1)(n-1)$ plus-edges between $V\left(M^{+}\right)$and $V\left(M^{-}\right)$. Consequently, there are exactly $\frac{1}{2} \cdot(2 n+2)(2 n-2)=2(n+1)(n-1)$ minus-edges between $V\left(M^{+}\right)$and $V\left(M^{-}\right)$.

Next, suppose, for a contradiction, that the number of plus-edges between vertices in $V\left(M^{-}\right)$is odd. In this case, there are two edges $e_{1}$ and $e_{2}$ in $M^{-}$and two disjoint edges $f_{1}$ and $f_{2}$ between $e_{1}$ and $e_{2}$ such that $f_{1}$ is a plus-edge and $f_{2}$ is a minus-edge. By Claim 6 and Claim 7, there are two edges $e_{3}$ and $e_{4}$ in $M^{+}$and two disjoint minus-edges $f_{3}$ and $f_{4}$ between $e_{3}$ and $e_{4}$. Again, the perfect matching $N=\left(M \backslash\left\{e_{1}, e_{2}, e_{3}, e_{4}\right\}\right) \cup\left\{f_{1}, f_{2}, f_{3}, f_{4}\right\}$ satisfies $\sigma(N)=\sigma(M)-2=0$, contradicting the choice of $M$. Hence, the number of plus-edges between vertices in $V\left(M^{-}\right)$is even, say equal to $2 k$ for some integer $k$. By (1), the number of minus-edges between vertices in $V\left(M^{+}\right)$is

$$
\left(4 n^{2}-n\right)-2(n+1)(n-1)-\left(\left(\begin{array}{c}
2 n-2 \\
2
\end{array}\right)-2 k\right)=4 n+2 k-1
$$

which is odd. Nevertheless, by Claim 7, the number of minus-edges between vertices in $V\left(M^{+}\right)$is even, which is a contradiction, and completes the proof.

The following is based on a construction given at the end of [2].

Proof of Proposition 2. Let $n$ be such that there exists a positive even integer $k$ with $4 n=k^{2}+4$. Let $(A, B)$ be a partition of the vertex set of $K_{4 n}$ with $|A|=\frac{1}{2}\left(k^{2}+k\right)+2$ and $|B|=\frac{1}{2}\left(k^{2}-k\right)+2$. Now, we define a function $\sigma: E\left(K_{4 n}\right) \rightarrow\{-1,1\}$ such that all edges between $A$ and $B$ receive the value 1 and all remaining edges receive the value -1 . Note that

$$
\begin{aligned}
\left|\sigma^{-1}(1)\right| & =|A| \cdot|B|=\left(\frac{k^{2}+k}{2}+2\right)\left(\frac{k^{2}-k}{2}+2\right)=\frac{\left(k^{2}+4\right)\left(k^{2}+3\right)+4}{4} \\
& =\frac{1}{2}\left(\begin{array}{c}
4 n \\
2
\end{array}\right)+1
\end{aligned}
$$

and thus,

$$
\sigma\left(E\left(K_{4 n}\right)\right)=\left|\sigma^{-1}(1)\right|-\left|\sigma^{-1}(-1)\right|=\left(\frac{1}{2}\left(\begin{array}{c}
4 n \\
2
\end{array}\right)+1\right)-\left(\frac{1}{2}\left(\begin{array}{c}
4 n \\
2
\end{array}\right)-1\right)=2 .
$$


Now, suppose, for a contradiction, that $M$ is a perfect matching in $K_{4 n}$ with $\sigma(M)=0$. Clearly, $M$ contains $n$ plus-edges between $A$ and $B$. Hence, the number of vertices in $A$ that are not covered by a plus-edge in $M$ is

$$
|A|-n=\left(\frac{k^{2}+k}{2}+2\right)-\frac{k^{2}+4}{4}=\frac{k}{2}\left(\frac{k}{2}+1\right)+1
$$

Since this is an odd number, by construction, not all these vertices can be covered by minus-edges in $M$, which is a contradiction, and completes the proof.

For the proof of our second main result, Theorem 3, we need the following extremal result about matchings due to Erdős and Gallai [5].

Theorem 10 (Erdős and Gallai [5]). If $G$ is a graph of order $4 n$ with matching number $n-k$ for some positive integer $k$, then the number of edges of $G$ is at most $\left(\begin{array}{c}4 n \\ 2\end{array}\right)-\left(\begin{array}{c}3 n+k \\ 2\end{array}\right)$, with equality if and only if $G$ is the complement of the disjoint union of a complete graph of order $3 n+k$ and $n-k$ isolated vertices.

We proceed to the proof of our second main result.

Proof of Theorem 3. Let $\sigma: E\left(K_{4 n}\right) \rightarrow\{-1,1\}$ be such that $\sigma\left(E\left(K_{4 n}\right)\right) \geqslant 0$ and such that $|\sigma(M)| \geqslant 2 k \geqslant 4$ for every perfect matching $M$ in $K_{4 n}$. As observed above, $|\sigma(M)|$ is even for every perfect matching $M$ in $K_{4 n}$. Therefore, it remains to show that $\sigma\left(E\left(K_{4 n}\right)\right)$ is at least the term stated in the theorem.

We distinguish the following two cases.

Case 1. $\sigma(M) \leqslant 0$ for some perfect matching $M$ in $K_{4 n}$.

We choose a perfect matching $M$ in $K_{4 n}$ with $\sigma(M) \leqslant 0$ such that $\sigma(M)$ is as large as possible. Since $\sigma(M) \leqslant-2 k \leqslant-4$, we obtain $\left|M^{-}\right| \geqslant n+k$ and $\left|M^{+}\right| \leqslant n-k$, where we use the notation and terminology as in the proof of Theorem 1. The following two observations correspond to Claim 5 and Claim 6 within the proof of Theorem 1.

If there is an edge $e$ in $M^{+}$and an edge $f$ in $M^{-}$such that there are two disjoint plus-edges $e^{\prime}$ and $f^{\prime}$ between $e$ and $f$, then the perfect matching $N=(M \backslash\{e, f\}) \cup\left\{e^{\prime}, f^{\prime}\right\}$ satisfies $\sigma(M)<\sigma(N)=\sigma(M)+2<0$, contradicting the choice of $M$. Hence, between every edge in $M^{+}$and every edge in $M^{-}$, there are at least two minus-edges, which implies that at least half the edges between $V\left(M^{+}\right)$and $V\left(M^{-}\right)$are minus-edges.

If there is no plus-edge between vertices in $V\left(M^{-}\right)$, then there are strictly more minusedges than plus-edges, contradicting $\sigma\left(E\left(K_{4 n}\right)\right) \geqslant 0$. Hence, there are two edges $e$ and $f$ in $M^{-}$such that there exists a plus-edge $e^{\prime}$ between $e$ and $f$. Let $f^{\prime}$ be the edge between $e$ and $f$ that is disjoint from $e^{\prime}$. The perfect matching $N=(M \backslash\{e, f\}) \cup\left\{e^{\prime}, f^{\prime}\right\}$ satisfies $\sigma(M)<\sigma(N) \leqslant \sigma(M)+4 \leqslant-2 k+4 \leqslant 0$, contradicting the choice of $M$. This contradiction completes the proof in this case.

Case 2. $\sigma(M)>0$ for every perfect matching $M$ in $K_{4 n}$.

Note that $\sigma(M) \geqslant 2 k$ for every perfect matching $M$ in $K_{4 n}$. Let $\nu$ be the matching number of the graph $G=\left(V\left(K_{4 n}\right), \sigma^{-1}(-1)\right)$. 
Suppose, for a contradiction, that $\nu>n-k$. If $M$ is a maximum matching in $G$, then $V(G) \backslash V\left(M^{-}\right)$is an independent set in $G$. This implies that there is a matching $M^{+}$in $\left(V\left(K_{4 n}\right), \sigma^{-1}(1)\right)$ covering all vertices in $V(G) \backslash V\left(M^{-}\right)$. Note that $\left|M^{+}\right|=2 n-\nu<n+k$, and, hence, $M^{-} \cup M^{+}$is a perfect matching in $K_{4 n}$ with $\sigma\left(M^{-} \cup M^{+}\right)<-(n-k)+(n+k)=$ $2 k$, which is a contradiction. Hence, we have $\nu \leqslant n-k$.

Let $\nu=n-k^{\prime}$ for some integer $k^{\prime} \geqslant k$. By Theorem 10, we obtain

$$
\begin{aligned}
\sigma\left(E\left(K_{4 n}\right)\right) & =\left(\begin{array}{c}
4 n \\
2
\end{array}\right)-2 m(G) \\
& \geqslant\left(\begin{array}{c}
4 n \\
2
\end{array}\right)-2\left(\left(\begin{array}{c}
4 n \\
2
\end{array}\right)-\left(\begin{array}{c}
3 n+k^{\prime} \\
2
\end{array}\right)\right) \\
& =2\left(\begin{array}{c}
3 n+k^{\prime} \\
2
\end{array}\right)-\left(\begin{array}{c}
4 n \\
2
\end{array}\right) \\
& \geqslant 2\left(\begin{array}{c}
3 n+k \\
2
\end{array}\right)-\left(\begin{array}{c}
4 n \\
2
\end{array}\right) \\
& =n(n-1)+k(6 n-1)+k^{2},
\end{aligned}
$$

which completes the proof.

\section{References}

[1] Y. Caro. Zero-sum problems - a survey. Discrete Math., 152:93-113, 1996.

[2] Y. Caro, A. Hansberg, J. Lauri, and C. Zarb. On zero-sum spanning trees and zerosum connectivity. arXiv:2007.08240v1, 2020.

[3] Y. Caro, A. Hansberg, and A. Montejano. Graphs isomorphisms under edgereplacements and the family of amoebas. arXiv:2007.11769, 2020.

[4] Y. Caro and R. Yuster. On zero-sum and almost zero-sum subgraphs over $\mathbb{Z}$. Graphs Comb., 32:49-63, 2016.

[5] P. Erdős and T. Gallai. On maximal paths and circuits of graphs. Acta Math. Acad. Sci. Hung., 10:337-356, 1959. 